\title{
PERFIL CONDICIONAL Y DE COMPOSICIÓN CORPORAL DE LOS PATINADORES DE VELOCIDAD DE ASODEPA BOGOTÁ, D.C.
}

CONDITIONAL PROFILE AND BODY COMPOSITION OF ASODEPA BOGOTA, D.C., ROLLER SPEED SKATERS.

\section{David Felipe Fonseca Galeano}

Profesional Universitario en Ciencias del Deporte de la Universidad de Ciencias Aplicadas y Ambientales.

E-mail:dafonseca@udca.edu.co

\section{José Luís Ramírez Antolínez}

Estudiante de Ciencias del Deporte de la Universidad de Ciencias Aplicadas y Ambientales.

E-mail: joseluiss07@hotmail.com

Jeansy Alonso Rodríguez Buitrago

Magister en Pedagogía de la Cultura Física, Especialista en Biometodología del Entrenamiento Deportivo y Docente Investigador de la Universidad de Ciencias Aplicadas y Ambientales.

E-mail:alonrod29@hotmail.com

\section{RESUMEN}

Dentro del estudio realizado con los patinadores de ASODEPA Bogotá (Asociación de Entrenadores de Patinaje), se pretendía determinar el perfil condicional y de composición corporal de este grupo de deportistas, por medio de bioimpedancia eléctrica, método de fácil manejo, económico y rápido, por otra parte, se aplicaron diferentes test de campo para evaluar las características físicas. Se realizó un estudio descriptivo, de enfoque cuantitativo con un diseño transversal, en una muestra total de 24 patinadores de velocidad sobre ruedas, 21 mujeres y 3 hombres, que cumplieron con los criterios de inclusión y exclusión previamente establecidos. Dentro de los resultados de las pruebas físicas, los deportistas se encuentran dentro de los rangos de bueno y excelente según las tablas de referencia establecidas, excepto en las pruebas de dinamometría manual en que los resultados no fueron los mejores, clasificándolos de forma no adecuada y pertinente. Para la composición corporal se analizó la talla, el peso y la edad frente a investigaciones nacionales e internacionales, presentando como resultado que los patinadores de ASODEPA Bogotá, D.C.M cuentan con los menores valores de cada ítem analizado, por otra parte, se compararon los porcentajes de grasa y masa magra, concluyendo que los hombres presentan un mayor porcentaje de masa magra y menor porcentaje de masa grasa, mientras que en mujeres un mayor porcentaje graso y menor masa magra, se concluyó que los deportistas de ASODEPA Bogotá se encuentran fuera de los rangos óptimos para un patinador de velocidad previamente estipulados, pero, debido a su edad, estos rangos pueden ser alcanzados de forma que sus valoraciones futuras sean lo más cercanas a las de un patinador élite de velocidad sobre ruedas.

Palabras clave: Composición corporal, bioimpedancia eléctrica, perfil condicional, patinaje de velocidad. 


\section{ABSTRACT}

In this study with ASODEPA roller skaters from Bogota (Skating Coaches Association), it was intended to determine the conditional profile and body composition of this group of athletes, by means of electric bioimpedance, easy to use, economical and fast, On the other hand, different field tests were applied to evaluate the physical characteristics. A descriptive study was carried out, with a quantitative approach with a transversal design, in a total sample of 24 roller speed skaters, 21 women and 3 men, who met the previously established inclusion and exclusion criteria. Within the results of the physical tests, the athletes are within the ranges of good and excellent according to the established reference tables, except in manual dynamometry tests in which the results were not the best, classifying them badly. Body composition was analyzed for size, weight and age compared to national and international research, showing that the ASODEPA Bogota roller skaters had the lowest values of each item analyzed, on the other hand, the percentages of fat were compared and muscle mass, concluding that men present a higher percentage of muscle mass and lower percentage of fat mass, while in women a higher fat percentage and lower muscle mass, it was concluded that ASODEPA Bogota athletes are away from the optimal ranges for a speed skater previously stipulated, but due to their age, these ranges can be reached so that their future ratings are as close as an elite speed roller skaters.

Key words: Body composition, electric bioimpedance, conditional profile, speed roller skate.

\section{INTRODUCCIÓN}

Era para Colombia su décimo cuarto título mundial de patinaje de velocidad sobre ruedas, se logró tras conseguir un total de veinte (20) medallas de Oro, Dieciséis (16) de plata y nueve (9) de bronce, precisamente, en el año 2016 en la ciudad de Nanjing, China, (FEDEPATIN, 2017). Esto describe un panorama prominente para el patinaje colombiano en el escenario internacional, debido a la conquista de títulos tanto en categorías Senior como en las categorías de base, masculino y femenino. Mantener esta posición de liderazgo requiere un trabajo riguroso con dedicación, esfuerzo y compromiso, fundamentados sobre cimientos investigativos como deportivos como una fuente de fortalecimiento hacia los programas de detección y promoción de talentos.

La selección de jóvenes talentos en el patinaje permite la formación de reservas deportivas capaces de suplir los equipos de categorías avanzadas en las principales competiciones nacionales e internacionales. Sin embargo, es necesario establecer indicadores de desempeño para guiar el proceso de selección de los deportes.

El análisis de la composición corporal permite conocer las proporciones de los distintos elementos del cuerpo humano y su estudio constituye el eje central de la valoración del estado nutricional (Sánchez \& Barón, 2009), dentro de estos componentes, se tiene en cuenta la edad, la talla, el sexo, el peso, el IMC y situación de sedentarismo del sujeto, uno de los métodos utilizados para evaluar estas características, es la bioimpedancia eléctrica, que es una técnica no invasiva, poco costosa y de fácil manejo, que permite el analizar como evaluar detalladamente la composición corporal 
humana, con estimaciones en cuanto a: tejido magro, tejido adiposo, hidratación y generar un dictamen del estado de salud del evaluado, complementando así, una parte importante de la evaluación clínica (Árias, 2010).

Como instrumento para la evaluación de la condición física de los deportistas, se ha optado por aplicar los test de campo, estos se pueden definir como tareas previamente estandarizadas, sujetas a determinadas leyes estadístico-matemáticas y son aplicadas con el objetivo de determinar las particularidades motoras y otros aspectos de la personalidad del ser humano (Matveev, citado por Pérez, 2008).

Es común utilizar estos estudios para generar un perfil auto-comparativo de los deportistas a lo largo de una línea de tiempo, o simplemente, en realizar un análisis comparativo de puntos referentes frente a modelos de deportistas competitivos nacionales e internacionales, previamente evaluados, además forja una expansión del deporte de forma teórica, fundamentando la investigación y el intercambio cultural deportivo. Así mismo, estos datos son utilizados para la selección deportiva, debido a que es un proceso a través del cual se individualiza a personas dotadas de talento y actitudes favorables para un deporte, con la ayuda de métodos y test científicamente válidos (Nadori, citado por Bermúdez, 2010). En el caso del alto rendimiento, se pueden tener en cuenta estudios previamente realizados sobre evaluaciones en deportistas que han ofrecido grandes logros competitivos, con el fin, de integrar una caracterización enfocada en capacidades condicionales y de composición corporal, que puede contribuir a una estandarización de un biotipo cercano a lo ideal en el patinaje de velocidad sobre ruedas, con objetivos puntuales como el mejoramiento en el rendimiento competitivo. En este caso, para el patinaje local, sería de gran aporte generar un perfil de composición corporal y condicional del grupo de velocistas de ASODEPA, quienes participan en competencias a nivel nacional, consideran ahora que este modelo podría ser tomado en cuenta por clubes o ligas de patinaje del país y a lo mejor pueda ir creciendo con estudios similares e ir formando el perfil del patinador de velocidad en el país, con el fin de continuar por la senda ganadora que hasta el momento el patinaje de velocidad ha forjado para Colombia.

\section{CAPÍTULO 1. ANTECEDENTES TEÓRICOS Y METODOLÓGICOS ACERCA DEL PERFIL CONDICIONAL Y DE COMPOSICIÓN CORPORAL EN PATINADORES SOBRE RUEDAS.}

De acuerdo a los objetivos planteados en esta investigación se realizó la revisión de literatura, abordando el tema del patinaje de velocidad sobre ruedas, y los fundamentos teóricos sobre composición corporal y características del perfil corporal de los deportistas.

\section{Antecedentes sobre el perfil condicional y de composición corporal en patinadores sobre ruedas.}

Acero (2002), tiene en cuenta la clasificación para hombres deportistas y no deportistas, de acuerdo a su porcentaje de masa grasa, elaborada por el Comité Olimpico de Estados Unidos en 1998, esta se puede emplear para realizar una comparación con los patinadores de velocidad de ASODEPA Bogotá, teniendo 
presente que los parámetros de edad de la muestra en tal estudio no fueron clasificados por categorías, lo que permitiría arribar a una conclusion más precisa.

\begin{tabular}{|c|c|c|c|c|}
\hline & Hombres & $\begin{array}{c}\text { Hombres } \\
\text { deportista de alto } \\
\text { rendimiento }\end{array}$ & Mujeres & $\begin{array}{c}\text { Mujeres } \\
\text { deportistas de } \\
\text { alto rendimiento }\end{array}$ \\
\hline $\begin{array}{c}\% \text { Masa } \\
\text { Grasa }\end{array}$ & $13 \%-15 \%$ & $4 \%-10 \%$ & $23 \%-25 \%$ & $10 \%-18 \%$ \\
\hline
\end{tabular}

Tabla 1: Rangos de porcentajes de grasa según Comité olímpico Estados Unidos 1998. Fuente: (Acero, 2002).

Además, durante la $1^{\text {a }}$ Copa del mundo de patinaje, la cual se realizó en Santa Fe de Bogotá en 1997, se logró estudiar y generar un promedio general de los participantes mundialistas en cada una de sus respectivas modalidades (Lozano, Contreras, \& Navarro, 2006), debido a estos dos estudios se evidencian los cambios que se generan en la caracterización del velocista de patinaje durante el transcurso del tiempo, reduciendo los valores de porcentaje de grasa corporal que pueden llegar a presentar los deportistas de alto rendimiento. En la tabla siguiente se presentan los datos al respecto:

\begin{tabular}{|c|c|c|c|c|}
\hline & $\begin{array}{c}\text { Hombres } \\
\text { Velocidad }\end{array}$ & Hombres Fondo & $\begin{array}{c}\text { Mujeres } \\
\text { Velocidad }\end{array}$ & Mujeres Fondo \\
\hline $\begin{array}{c}\text { \% Masa } \\
\text { Grasa }\end{array}$ & $5,3 \%$ & $6,9 \%$ & $6,9 \%$ & $6,8 \%$ \\
\hline
\end{tabular}

Tabla 2: Rangos de promedios de porcentajes de grasa. Fuente: (Lozano, Contreras, \& Navarro, 2006).

Un primer estudio corresponde al realizado por Lozano, Contreras, \& Navarro (2006), titulado: “Descripción antropométrica de los patinadores de velocidad sobre ruedas participantes en los Juegos Deportivos Nacionales de Venezuela", este estudio tuvo por objetivo generar un perfil a partir de características físicas de un grupo de patinadores de velocidad compuesto por 81 deportistas (48 hombres y 33 mujeres), participantes en los Juegos Deportivos Nacionales de Venezuela Andes, diciembre de 2005, en la ciudad de San Antonio del Táchira. Se aplicó la técnica de medición antropométrica sugerida por el "ISAK", calculando:

- Porcentaje de grasa corporal (Yuhasz, 1974).

- Composición corporal ( (Mazza, 2000). 
- Somatotipo, de acuerdo al metodo Heath and Carter (1980).

Para el análisis estadístico se utilizó el software SPSS 7.5, con un nivel de confianza del $95 \%$.
Los valores de referencia se organizaron de acuerdo a la modalidad deportiva y por sexo:

\begin{tabular}{|l|c|l|c|l|}
\hline \multicolumn{1}{|c|}{ Especialidad } & $N^{\mathbf{0}}$ & Edad (años) & Peso (Kg.) & \multicolumn{1}{c|}{ Talla (cm.) } \\
\hline Hombres Velocidad & 19 & $18,56 \pm 2,69$ & $64,14 \pm 3,95$ & $171,3 \pm 3,95$ \\
\hline Hombres Fondo & 29 & $19,66 \pm 2,73$ & $61,93 \pm 6,19$ & $170,6 \pm 6,36$ \\
\hline Mujeres Velocidad & 15 & $17,27 \pm 2,21$ & $55,05 \pm 7,22$ & $158,36 \pm 7,39$ \\
\hline Mujeres Fondo & 18 & $19,15 \pm 2,48$ & $55,25 \pm 5,66$ & $163,91 \pm 5,86$ \\
\hline
\end{tabular}

Tabla 3: Organización por género y especialidad deportiva. Fuente: (Lozano, Contreras, \& Navarro, 2006).

De igual manera, en el estudio se organizó la información respecto a la composición corporal de los patinadores, realizando la comparación entre cada una de las especializaciones deportivas y por sexo.

\begin{tabular}{|l|l|l|l|l|}
\hline $\begin{array}{l}\text { Especialidad / } \\
\text { Composición Corporal }\end{array}$ & $\begin{array}{l}\text { Velocidad } \\
\text { Hombres }\end{array}$ & $\begin{array}{l}\text { Fondo } \\
\text { Hombres }\end{array}$ & $\begin{array}{l}\text { Velocidad } \\
\text { Mujeres }\end{array}$ & $\begin{array}{l}\text { Fondo } \\
\text { Mujeres }\end{array}$ \\
\hline \% Graso: & $9,59 \pm 1,17$ & $9,65 \pm 1,87$ & $19,59 \pm 2,06$ & $18,43 \pm 2,18$ \\
\hline Peso Graso (Kg.): & $6,17 \pm 0,95$ & $6,01 \pm 1,45$ & $10,85 \pm 2,10$ & $10,19 \pm 1,63$ \\
\hline$\%$ Óseo: & $17,11 \pm 1,34$ & $17,6 \pm 1,55$ & $15,71 \pm 1,52$ & $16,12 \pm 0,91$ \\
\hline Peso Óseo (Kg.): & $10,97 \pm 1,03$ & $10,85 \pm 1$ & $8,60 \pm 0,98$ & $8,89 \pm 0,91$ \\
\hline \% Muscular: & $49,19 \pm 1,72$ & $48,66 \pm 1,74$ & $43,79 \pm 2,12$ & $44,55 \pm 2,05$ \\
\hline Peso Muscular (Kg.): & $31,54 \pm 2,09$ & $30,16 \pm 3,35$ & $24,11 \pm 3,33$ & $24,62 \pm 2,07$ \\
\hline$\%$ Residual: & $24,1 \pm 0$ & $24,1 \pm 0$ & $20,90 \pm 0$ & $20,90 \pm 0$ \\
\hline Peso Residual (Kg.): & $15,46 \pm 0,95$ & $14,93 \pm 1,49$ & $11,51 \pm 1,51$ & $11,55 \pm 1,18$ \\
\hline
\end{tabular}

Tabla 4: Organización por género y especialidad deportiva, composición corporal. Fuente: (Lozano, Contreras, \& Navarro, 2006).

Otro estudio realizado con datos de competidores mundiales (Sovak \& Hawes, citado por Burke, 2007), brindan una herramienta que permite realizar análisis y comparaciones en cuanto a edad, peso y talla de patinadores de velocidad, a la cual pertenecen mujeres con rangos de edad entre los 19 - 28 años, de talla entre de 1,63 -
1,75 metros y 57,3 - 75 kilogramos de peso. En esta investigación se emplea para comparar los velocistas colombianos con patinadores del contexto mundial y como guía para establecer los controles del entrenamiento respecto a las características referidas en este estudio. 


\begin{tabular}{|c|c|c|}
\hline \multicolumn{3}{|c|}{ (Sovak \& Hawes, 1987) } \\
\hline & Mujere s & Hombres \\
\hline Edad: & $19-28$ & $21-31$ \\
\hline Talla (m): & $1,63-1,75$ & $1,62-1,91$ \\
\hline Pes o (kg): & $57,3-75$ & $64,5-95,5$ \\
\hline
\end{tabular}

Tabla 5: Rangos ideales patinadores de velocidad. Fuente: (Burke, 2007).

Otro trabajo examinado es el de Matyk \& Raschka (2011), en Alemania, el objetivo principal consistía en valorar tanto por pliegues cutáneos como por bioimpedancia eléctrica a un total de 45 patinadores (26 hombres y 19 mujeres) y como segundo objetivo, realizar la respectiva comparación con un grupo control compuesto por 49 sujetos (28 hombres y 21 mujeres), los datos serían tratados a través de ANOVA.

\begin{tabular}{|l|c|c|c|c|c|c|c|}
\hline & $\begin{array}{c}\text { Long } \\
\text { Distance } \\
\text { male }\end{array}$ & $\begin{array}{c}\text { Short } \\
\text { Distance } \\
\text { male }\end{array}$ & $\begin{array}{c}\text { Control } \\
\text { group } \\
\text { male }\end{array}$ & $\begin{array}{c}\text { Long } \\
\text { Distance } \\
\text { female }\end{array}$ & $\begin{array}{c}\text { Short } \\
\text { Distance } \\
\text { female }\end{array}$ & $\begin{array}{c}\text { Control } \\
\text { group } \\
\text { female }\end{array}$ & p \\
\hline (n): & 12 & 14 & 28 & 11 & 8 & 21 & n.s. \\
\hline Age (yrs): & 21.7 & 23.0 & 21.3 & 23.0 & 21.6 & 19.5 & n.s. \\
& $(2.3)$ & $(3.9)$ & $(3.8)$ & $(2.7)$ & $(2.6)$ & $(2.9)$ & \\
\hline Height (cm): & 174.8 & 177.3 & 180.4 & 159.9 & 166.0 & 169.6 & n.s. \\
& $(5.6)$ & $(5.9)$ & $(5.9)$ & $(3.9)$ & $(5.0)$ & $(7.0)$ & \\
\hline Mass (kg): & 68.9 & 73.2 & 75.7 & 52.8 & 58.3 & 64.8 & $\leq 0.001$ \\
& $(6.3)$ & $(7.5)$ & $(10.0)$ & $(4.8)$ & $(2.1)$ & $(13.1)$ & \\
\hline BIA-Fat & 8.0 & 8.1 & 15.3 & 17.7 & 20.8 & 24.5 & n.s. \\
(\%): & $(1.9)$ & $(3.0)$ & $(6.9)$ & $(4.0)$ & $(2.3)$ & $(5.7)$ & \\
\hline Caliper-Fat & 11.0 & 9.7 & 17.8 & 17.5 & 17.6 & 24.8 & n.s. \\
(\%): & $(2.1)$ & $(2.0)$ & $(6.5)$ & $(2.2)$ & $(1.0)$ & $(4.9)$ & \\
\hline Broca Index: & 92.0 & 94.7 & 94.6 & 98.0 & 98.5 & 103.2 & n.s. \\
& $(5.4)$ & $(6.1)$ & $(14.2)$ & $(7.5)$ & $(6.1)$ & $(15.2)$ & \\
\hline BMI & 22.5 & 23.2 & 23.3 & 20.6 & 21.2 & 22.4 & n.s. \\
(kg/m $\left.{ }^{2}\right):$ & $(1.3)$ & $(1.5)$ & $(3.4)$ & $(1.5)$ & $(1.0)$ & $(3.4)$ & \\
\hline
\end{tabular}

Tabla 6: Organización datos de medición. Fuente: (Matyk \& Raschka, 2011).

Los resultados mostrados por medio de somatocarta (Heath and Carter) mostraron que los velocistas de sexo masculino tenían una mayor predisposición a ser ectomesomorfos. Para las mujeres velocistas la tendencia era mínima hacia lo endomesomorfo. Otro aspecto de interés de este estudio es que relaciona la diferencia que puede existir entre la medición por pliegues cutáneos y la realizada mediante la bioimpedancia.

También, se consultó el estudio de Lozano \& Cárdenas (2013), titulado: “Análisis de la composición corporal en la preparación de los patinadores de velocidad de la Selección Norte de Santander participantes en los 
Juegos Nacionales 2012", es una investigación de carácter descriptivo, en la cual se valoró un total de 14 deportistas (10 mujeres y 4 hombres) en las modalidades de patinaje sobre ruedas.

Para llevar a cabo las valoraciones hicieron uso del protocolo desarrollado por el Grupo Español de Cineantropometría (GREC) citado en Esparza (1993), así mismo utilizando las herramientas de determinación de porcentaje de masa grasa (Yuhasz, 1974), porcentaje de masa magra (Mazza, 2000) y el somatotipo de acuerdo al protocolo desarrollado por Heath Carter en 1975, con esta herramienta definirían el biotipo específico de acuerdo al sexo y la especialidad deportiva.

\begin{tabular}{|c|c|c|c|c|c|c|c|c|}
\hline \multirow{2}{*}{ Sujetos } & \multicolumn{2}{|c|}{ \% Graso } & \multicolumn{2}{c|}{ Peso \% Graso } & \multicolumn{2}{c|}{ \% Muscular } & \multicolumn{2}{c|}{ Peso \% Muscular } \\
\cline { 2 - 9 } & Promedio & D.E. & Promedio & D.E. & Promedio & D.E. & Promedio & D.E. \\
\hline $\begin{array}{c}\text { Mujeres } \\
\text { especialidad } \\
\text { velocidad (n=5): }\end{array}$ & 19,205 & 0,22 & 10,88 & 0,43 & 44,83 & 0,15 & 25,4 & 1,37 \\
\hline $\begin{array}{c}\text { Mujeres } \\
\text { especialidad } \\
\text { fondo (n=5): }\end{array}$ & 18,64 & 1,4 & 10,75 & 1,44 & 44,94 & 1,04 & 25,83 & 2,24 \\
\hline $\begin{array}{c}\text { Hombres } \\
\text { especialidad } \\
\text { velocidad (n=2): }\end{array}$ & 11,26 & 1,31 & 7,5 & 1,81 & 47,35 & 0,87 & 31,16 & 4,57 \\
\hline $\begin{array}{c}\text { Hombres } \\
\text { especialidad } \\
\text { fondo (n=2): }\end{array}$ & 10,48 & 1,02 & 6,43 & 0,72 & 49,06 & 0,12 & 30,05 & 0,4 \\
\hline
\end{tabular}

Tabla 7: Organización deportistas por especialidad deportiva. Fuente: (Lozano \& Cárdenas, 2013).

En el estudio se halló que para las mujeres velocistas predominó el componente endomórfico, mientras que para los hombres de la misma especialidad predominó el mesomórfico, incluso influyo en la especialidad de fondo.

En su trabajo investigativo, Lozano \& Cárdenas (2013), concluyen que los estudios de composición corporal son de suma importancia, porque brindan parámetros que fortalecen la determinación del rendimiento deportivo del evaluado según su especialidad deportiva.

Entre los estudios que permitan establecer la relación de perfil condicional, se encontró el de la Licenciada, Bertha Susana Paz Viteri
(2016), titulado: “La condición física en la aptitud deportiva del patinaje de la categoría infantil en la Federación Deportiva de Chimborazo." Cuyo principal objetivo es la caracterización de los patinadores categoría infantil de la Federación Deportiva de Chimborazo, en Ecuador. Este estudio brinda una herramienta de medición de campo, que se aplicó de igual manera a los patinadores de ASODEPA Bogotá, que es la dinamometría manual y la prueba de salto largo sin impulso.

La muestra del estudio es de 52 patinadores (36 niñas y 16 niños) en edades entre los 6-13 años, no se específica la modalidad de los patinadores, fueron valorados bajo los parámetros de la batería 
alpha fitness creado en España y validado en Latinoamérica en Colombia, se ejecutó mediante una pre toma de datos y al cabo de 10 semanas se volvió a medir a la población (Paz, 2016).
Las tablas siguientes muestran los resultados obtenidos del estudio realizado por Paz (2016).

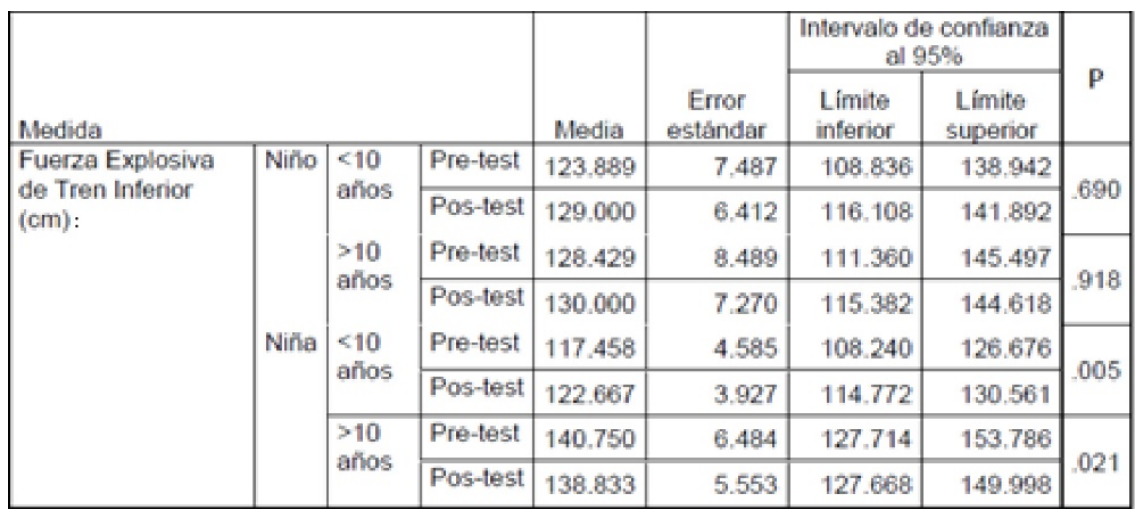

Tabla 8: Medida de fuerza de tren inferior (salto largo sin impulso). Fuente: (Paz, 2016).

\begin{tabular}{|c|c|c|c|c|c|c|c|}
\hline \multirow{2}{*}{\multicolumn{4}{|c|}{ Medida }} & \multirow{3}{*}{$\begin{array}{r}\text { Media } \\
9.204\end{array}$} & \multirow{3}{*}{$\begin{array}{r}\text { Error eständar } \\
.849 \\
\end{array}$} & \multicolumn{2}{|c|}{ Intervalo de confianza al $95 \%$} \\
\hline & & & & & & Límite inferior & Límite superior \\
\hline \multirow{8}{*}{$\begin{array}{l}\text { Fuerza Máxima de } \\
\text { Tren Superior }(\mathrm{kg}) \text { : }\end{array}$} & \multirow[t]{4}{*}{ Niño } & \multirow[t]{2}{*}{$<10$ años } & Pre-test & & & 7.496 & 10.911 \\
\hline & & & Pos-test & 10.756 & 995 & 8.755 & 12.756 \\
\hline & & \multirow[t]{2}{*}{$>10$ años } & Pre-test & 15.757 & 962 & 13.823 & 17.692 \\
\hline & & & Pos-test & 16.459 & 1.128 & 14.191 & 18.727 \\
\hline & \multirow[t]{4}{*}{ Nifía } & \multirow[t]{2}{*}{$<10$ aก็os } & Pro-test & 8.884 & 520 & 7.840 & 9.929 \\
\hline & & & Pos-test & 10.472 & 609 & 9247 & 11.697 \\
\hline & & \multirow[t]{2}{*}{$>10$ afios } & Pro-test & 16.283 & .735 & 14.806 & 17.761 \\
\hline & & & Pos-test & 16.458 & .862 & 14.726 & 18.191 \\
\hline
\end{tabular}

Tabla 9: Medida de fuerza de tren superior (dinamometría manual). Fuente: (Paz, 2016).

\section{Fundamentos teóricos acerca del perfil condicional y de composición corporal.}

El patinaje de carreras es uno de los deportes más representativos de nuestro país, el cual, nos ha dado reconocimiento a nivel internacional, cabe resaltar que surge a finales del siglo XX como una alternativa a partir del patinaje sobre hielo, debido a que los deportistas necesitaban un espacio donde se les permitiera entrenar en época de verano, Batalla y Martínez (2002).

La competencia de este deporte se puede realizar en dos terrenos, pista con una longitud de 200 metros y ruta con un recorrido de 400 metros, Grelon (1998). Las pruebas tienen una distancia que van desde 200 metros hasta maratón, donde se destacan las pruebas de velocidad, fondo y 
semifondo. Hay que tener en cuenta que el deportista debe superar dichas distancias en el menor tiempo posible Rangel (2012), Salvador (2012).

El patinaje puede ser practicado de forma recreativa o competitiva; pudiéndose iniciar la práctica desde los cinco años, logrando sus máximos resultados a los 22 años; en la medida que se consolidan sus resultados la exigencia crece necesitándose de una alta preparación física, técnica, táctica y psicológica, debido a que los sistemas energéticos se deben combinar partiendo de una disposición aeróbica, debido a que el consumo de oxígeno durante todas las pruebas es elevado. Además de la predisposición del organismo para realizar esfuerzos anaeróbicos dependiendo del desarrollo de la prueba Hawley\&Burke (2000), Granell \& Cervera (2003), González (2012).

\section{Composición corporal}

Dentro del estudio realizado con los patinadores de ASODEPA Bogotá, uno de los principales ítems de estudio es la composición corporal, que es definida como el análisis que permite conocer las proporciones de los distintos componentes del cuerpo humano y su estudio constituye el eje central de la valoración del estado nutricional (Sánchez \& Barón, 2009). Este tipo de análisis mide los niveles de obesidad que pudiese presentar una determinada población, más aún, es de vital importancia conocer los diferentes componentes que permiten una correcta caracterización de la composición corporal, como lo es: estimación de agua corporal total (ACT), estimación de la masa grasa (MG), estimación de la masa libre de grasa (MLG) y la estimación de la masa mineral ósea, hay que mencionar, además, que se deben conocer los niveles adecuados y pertinentes según la edad, en la cual no exista un exceso o una falta en alguno de estos componentes de la composición corporal, que pueden dar lugar a generar indicios de algún tipo de enfermedad crónica (Taylor, Jones, Williams, \& Goulding, 2000); (Bray, DeLany, Harsha, Volaufova, \& Champagne, 2001), citados por: (Aristizábal \& Restrepo, 2014).

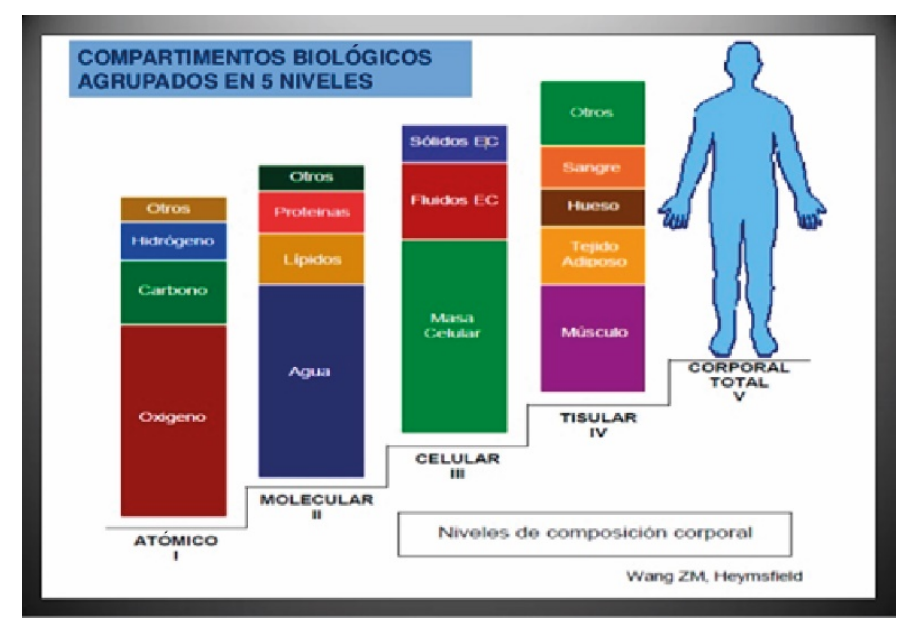

Imagen 1: Niveles de composición corporal. Fuente: https://es.slideshare.net/veronicadelgadolopez/composicion-corporal-1 
Heymsfield presentó el modelo de composición corporal, el cual, se compone de cinco niveles (Imagen 1), este consiste en más de 30 componente evaluables, organizados así: nivel atómico, nivel molecular, nivel celular, nivel tisular y organismo completo. Se cuenta con un gran número de técnicas y métodos para la medición y determinación de la composición corporal, así que, lo más conveniente es el uso combinado de las diferentes técnicas, método y equipos para conocer la composición corporal de un individuo (Suverza \& Haua, 2009).

En cuanto a la estimación de la composición corporal, el estudio de la morfología, las dimensiones y la proporcionalidad en relación al rendimiento deportivo, la nutrición y el crecimiento, todo este conjunto de elementos, está incluido en los objetivos de la cineantropometría (Alvero, y otros, 2009).

La cineantropometría permite evaluar la composición corporal, morfología, estado nutricional y proporciones de los deportistas, y con estos datos, se puede orientar hacia los parámetros adecuados y pertinentes para el rendimiento en su respectivo deporte. Se debe agregar que, también permite comprobar en deportistas jóvenes posibles desviaciones en su aparato locomotor, debido a los estudios de simetría en su desarrollo corporal (Nahrstaedt, Schauer, Shalaby, Hesse, \& Raisch, citado por Garcia, y otros, 2014).

Para valoración de los deportistas por medio de la Antropometría, se cuenta con métodos como la toma de pliegues de grasa o en este caso de estudio como la bioimpedancia.

\section{Bioimpedancia}

Se considera como un nuevo método de medición de la composición corporal, no invasivo, innovador, de fácil aplicación, de alguna forma un procedimiento atractivo, debido a que es sencillo, económico y rápido (Aristizábal \& Restrepo, 2014).

La bioimpedancia actúa en el cuerpo midiendo la resistencia al flujo en diversas regiones corporales de diferentes corrientes eléctricas. Se presentará una mayor resistencia al paso de la corriente en individuos con grandes cantidades de grasa, porque esta conduce débilmente. la electricidad, debido a que tiene poca cantidad de agua, mientras que en el músculo, que es un tejido bien hidratado, la conducción eléctrica será de mejor manera (Alvero, J. , citado por García, y otros, 2014).

El uso de la bioimpedancia eléctrica para estimar la composición corporal está basado en la consideración del cuerpo humano como un cilindro homogéneo y en las propiedades eléctricas de los tejidos corporales al paso de varias frecuencias de corrientes (únicas o múltiples). Los tejidos que contienen mucha agua y electrolitos, como el fluido cerebroespinal, la sangre o el músculo, son altamente conductores, mientras que la grasa, el hueso o los espacios con aire, como los pulmones, son tejidos altamente resistentes (Pietrobelli, Wang, \& Heymsfield, citado por Sánchez \& Barón, 2009).

Dentro de los parámetros para una buena medición por medio de bioimpedancia se encuentran: 
- No comer, ni beber en las 4 horas previas al test de bioimpedancia.

- No realizar ejercicio extenuante 12 horas antes.

- Orinar 30 min. antes del test.

- No consumir alcohol 48 horas antes.

- No tomar diuréticos 7 días antes.

- No realizar preferentemente la bioimpedancia en fase lútea (retención de líquidos).

- Retirar todo elemento metálico del cuerpo (relojes, anillos, pulseras, pendientes, piercings, entre otros) y no realizar el test sobre una camilla metálica. (Martínez \& Urdampilleta, 2012).

Con respecto a las diferentes publicaciones halladas para la respectiva comparación de los deportistas de ASODEPA Bogotá y sus puntos de evaluación en cuanto a la composición corporal, se recurrieron a tres estudios, pero, debido a la falta de investigaciones acerca del patinaje de velocidad sobre ruedas se tuvo que realizar la comparación frente a otros métodos de medición como es la toma de pliegues cutáneos, dentro de estos estudios tenemos los de (Lozano \& Cárdenas, 2013) y (Lozano, Contreras, \& Navarro, 2006).

Frente al estudio europeo de (Matyk \& Raschka, 2011), se generaron dos mediciones por la técnica de pliegues (no se especifica el método utilizado) y la segunda, fue por bioimpedancia, la comparativa de los dos métodos que se recopiló en la siguiente tabla: 


\begin{tabular}{|l|c|c|c|c|}
\hline \multicolumn{5}{|c|}{ PORCENTAJES DE GRASA } \\
\hline & \multicolumn{3}{|c|}{ Bioimpedancia } & \multicolumn{2}{c|}{ Pliegues cutáneos } \\
\hline & Promedio & Desviación estándar & Promedio & Desviación estándar \\
\hline Mujeres Velocidad: & 20,8 & 2,3 & 17,6 & 1 \\
\hline Hombres Velocidad: & 8,1 & 3 & 9,7 & 2 \\
\hline
\end{tabular}

Tabla 10: Comparación resultados porcentaje de grasa por bioimpedancia y pliegues cutáneos. Fuente: (Matyk \& Raschka, 2011)

De acuerdo a los valores anteriores, es válido reconocer que entre los métodos van a existir diferencias en los resultados obtenidos, ya sea por la validez y fiabilidad con la que cuente el método, en valoraciones de composición corporal, para (Aristizábal \& Restrepo, 2014), la bioimpedancia es un método de medición no invasivo, innovador, de fácil aplicación, un procedimiento atractivo, porque es fácil, económico y rápido en su aplicación.

Este método podría considerarse a la hora de una medición de un grupo grande y un espacio de tiempo reducido para la elaboración de los resultados. Sin embargo, el uso de pliegues cutáneos es el método preferido para examinar grandes grupos a expensas de bajos costos, sin el requerimiento de equipos de gran precisión, así mismo, presenta resultados con mejores correlaciones a comparación de otros métodos más precisos como la densitometría ósea (DEXA) y PH. (Nunes, Silva, Monteiro, \& Veras, 2001) Y (Lintsi, Kaarma, \& Kull, 2004), citados por (Ripka, Rotta, Ulbricht, \& Neves, 2014).

\section{CAPÍTULO 2. METODOLOGÍA RECOLECCIÓN DE DATOS Y MEDICIONES PATINADORES ASODEPA BOGOTÁ}

A continuación se expone la metodología empleada en la medición de los patinadores ASODEPA Bogotá, D.C..

\section{Tipo de estudio}

Se puntualiza como un estudio descriptivo de enfoque cuantitativo, debido a que, según (Hernández, Fernández, \& Baptista, 2010), se recolectan datos o componentes sobre diferentes aspectos del personal de la organización, en este caso, los deportistas pertenecientes a ASODEPA Bogotá y se realiza un análisis y medición de los mismos.

"La investigación descriptiva busca especificar propiedades, características y rasgos importantes de cualquier fenómeno que se analice" (Hernández, Fernández, \& Baptista, 2010). 
De igual manera, se caracteriza por contar con un diseño transversal, porque se recolectan datos en un solo momento, en un tiempo único, con un propósito de describir variables y analizar su incidencia e interrelación en un momento dado (Hernández, Fernández, \& Baptista, 2010).

\section{Población}

Se tomó como población a los patinadores de velocidad de los clubes pertenecientes a ASODEPA Bogotá, quienes realizan su preparación enfocados en diferentes torneos locales, departamentales o nacionales, con entrenamientos a doble jornada, donde en la mañana realizan la rutina de gimnasio, y en las tardes, el entrenamiento se orienta en la parte técnica durante dos horas y media.

\section{Muestra}

La muestra está constituida por un total de 24 patinadores (Mujeres: 21; Hombres: 3), cumplieron con los requisitos de inclusión para la realización del estudio. Todos los sujetos fueron informados acerca de los procedimientos a los que se someterían y firmaron un documento de consentimiento informado con anterioridad.

\section{Procedimiento para las mediciones}

Para la toma de los datos de composición corporal y test de campo en los patinadores de velocidad de ASODEPA, se desarrollaron en varias fases, que tenían una duración de un (1) día, así mismo se facilitaba la toma y control de los datos obtenidos, se siguió el siguiente proceso:

\section{Fase informativa:}

- Esta fase se ejecutó en un único día y se inició con la presentación del proyecto a los clubes pertenecientes a ASODEPA, los padres de familia o representantes legales de cada uno de los patinadores.

- Publicación de las fechas de las jornadas de medición antropométrica y aplicación de los test de campo ante los padres de familia y representantes legales, así mismo, notificar acerca de los requisitos y recomendaciones para cada una de las pruebas.

- Diligenciamiento del consentimiento informado por parte de los padres de familia o representante legal de los deportistas voluntarios para el estudio.

\section{Fase de medición de la composición corporal:}

- De acuerdo a las recomendaciones para una óptima medición de composición corporal por medio de bioimpedancia eléctrica (p. 15), se les recordó tanto a los deportistas como a los padres de familia o representante legal, dos días antes de las mediciones.

- Se generó la respectiva base de datos básicos (Nombre completo, Edad, Sexo, Estatura, Peso e IMC), también, de las referencias de composición corporal proporcionados por la Báscula TANITA (Porcentajes de grasa, agua, grasa visceral y muscular, perímetros de brazo izquierdo y derecho, edad metabólica y calorías) de los deportistas participantes.

- Se realizó la respectiva medición de la composición corporal de los sujetos 
por medio de Bioimpedancia eléctrica, se ejecutó en un único día.

\section{Fase de ejecución de test de campo:}

- Se generaron las bases de datos para los test a aplicar y la digitalización ordenada de la información en Excel.

- Exposición y Ejecución de las diferentes baterías de test distribuidos por estaciones así:

1. Test 1: Tapping-test de manos.

2. Test 2: Tapping-test de pies.

3. Test 3: Test de bastón de Galton.

4. Test 4: Test de salto horizontal sin impulso.

5. Test 5: Test de dinamometría manual.

6. Test 6: Carrera de 20 metros y 30 metros planos.

\section{Fase de medición $\mathrm{VO}_{2}$ máx.:}

- El test de Luc Legger se realizó en un único día, debido a que es un test de gran esfuerzo físico, se cumplió con la explicación del protocolo y sus recomendaciones, iniciando con un calentamiento previo con el objetivo de elevar la temperatura corporal y el ritmo cardiaco de los evaluados. De igual manera, a cada deportista se le generó su registro de datos al momento en que finalizaba la prueba.

\section{Fase de organización de datos:}

- En este período se realizó la organización de la información, con la ayuda del software Excel, se realizó la base de datos con los deportistas que cumplieran con la presentación de todas las pruebas físicas que anteriormente se habían expuesto, la no presentación de una sola prueba, los excluía automáticamente del estudio siguiendo los criterios de inclusión y exclusión del presente estudio.

- Como herramienta para el análisis estadístico se trabajó con el software InfoStat, que es un programa computacional que cubre tareas básicas de estadística descriptiva, generando las tablas ordenadas con las respectivas medidas de tendencia central.

\section{Fase de análisis de resultados:}

- Se realizó la comparación de los datos de los deportistas frente a estudios relacionados y previamente consultados, para constituir una evaluación a los procesos que se han venido gestionando con cada uno de los patinadores participes en este estudio y así brindar un panorama del patinaje de velocidad a nivel local e internacional.

DISCUSIÓN:

COMPARATIVA DE RESULTADOS

PATINADORES ASODEPA FRENTE A ESTUDIOS RELACIONADOS

A continuación se realizó la comparación de los Patinadores de velocidad de ASODEPA frente a estudios nacionales e internacionales realizados siguiendo parámetros evaluativos similares:

De acuerdo, a los promedios de edad de los participantes, en la gráfica 4 se puede determinar que la muestra más joven fue la del estudio con los deportistas de ASODEPA Bogotá, obteniendo en las mujeres una diferencia del $3.75 \%$ y en hombres un $18.68 \%$ con respecto al estudio de (Lozano \& Cárdenas, 2013). 


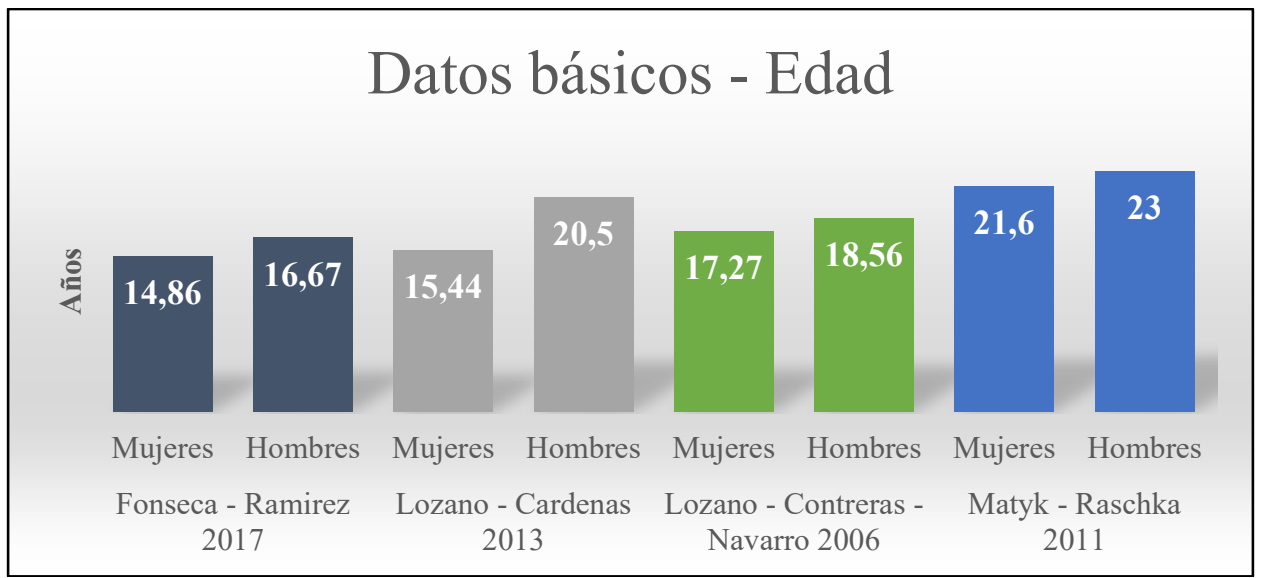

Gráfica 4: Comparación estudios - Datos básicos - Edad. Fuente: Elaboración propia.

Para la comparación respecto a la publicación de (Lozano, Contreras, \& Navarro, 2006), para mujeres, existió una diferencia del $13.95 \%$, mientras que, para los hombres una diferencia del $10.18 \%$.

Ahora bien, respecto a la muestra de patinadores de velocidad europeos (Matyk \& Raschka, 2011), la diferencia de edad es mucho mayor (Mujeres: 31.20\%; Hombres: $27.52 \%)$, debido a que la muestra se encuentra dentro de competiciones de élite, así mismo, obedece a los rangos de edad óptimos para un patinador de velocidad (Tabla 5).

Para los parámetros de la talla, la gráfica 5 muestra que ASODEPA Bogotá, conserva los valores más bajos frente a los demás estudios, con una diferencia del $6.02 \%$ para mujeres y del $1.79 \%$ para hombres, en comparativa con el estudio realizado por (Lozano \& Cárdenas, 2013).

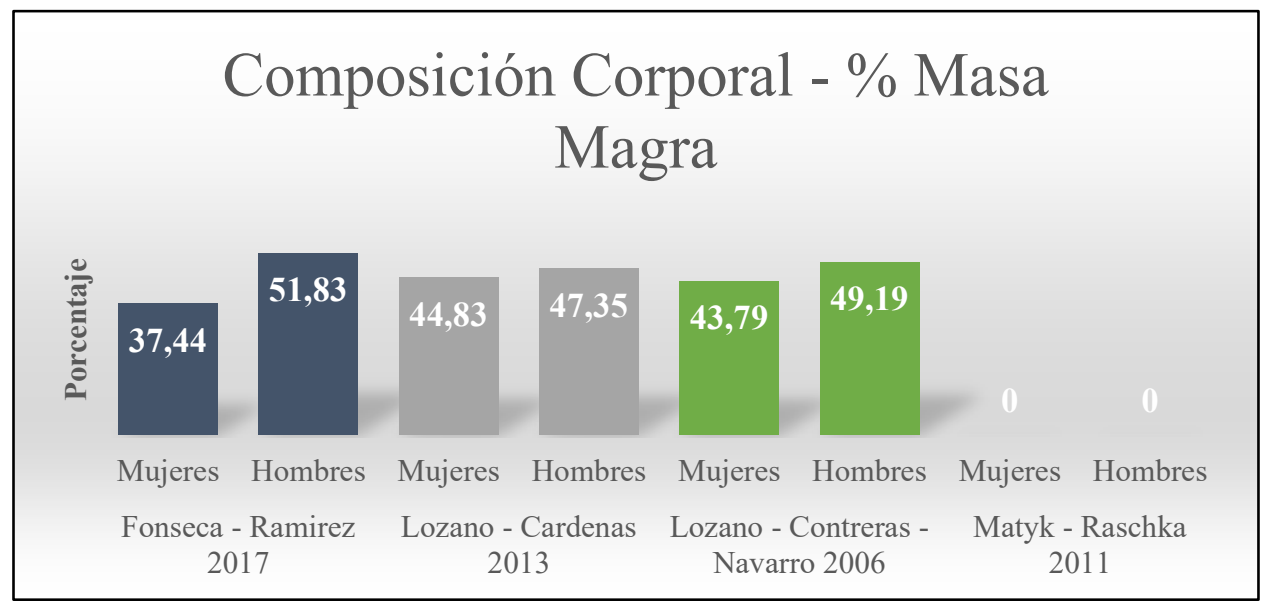

Gráfica 8: Comparación estudios - Composición corporal - Porcentaje masa magra. Fuente: Elaboración propia. 
Para la comparación respecto a la publicación de (Lozano, Contreras, \& Navarro, 2006), para mujeres, continua siendo el más bajo, manifestando una diferencia de $14.50 \%$, mientras que, para los hombres una diferencia de $5.09 \%$.

Por otra parte, respecto a la muestra de patinadores de velocidad europeos, (Matyk \& Raschka, 2011), no la tuvieron presente a la hora de la valoración antropométrica de sus deportistas.

Para el salto largo se realizó la evaluación frente a antecedentes encontrados realizados por diferentes autores, como es el realizado por (Paz, 2016), realizando la comparación de los resultados del test de salto largo sin impulso se observa que el promedio para niñas mayores de 10 años es de: $1,40 \mathrm{~m}$. mientras que el de las patinadoras de ASODEPA es de $1,72 \mathrm{~m}$. generando así, una diferencia de 18,60\%, vale la pena resaltar que ( $\mathrm{Paz}, 2016)$, no deja en claro los rangos de edades trabajados en el estudio, únicamente, nombra mayores y menores de 10 años.

En las categorías femeninas de ASODEPA los resultados de la medición generaron una calificación excelente de acuerdo a las tablas de referencia (Tabla 21), con los siguientes promedios: Infantil: 1,67 m.; Pre-Juvenil: 1,7 m. y Juvenil: 1,81 m. Para el caso de los hombres, se presenta un promedio de 2,24 $\mathrm{m}$. siendo esta una calificación excelente de acuerdo a la tabla 20 y una diferencia del $42,85 \%$ igualmente, no se específica un rango límite de edad, por tal motivo, la diferencia es grande porque los varones de ASODEPA cuentan con un promedio de edad de 16,67 años.

De igual manera, se comparó la dinamometría manual con el estudio de (Paz, 2016), generando una diferencia del 25,21\% respecto a los promedio de (Paz, 2016), para niñas mayores de 10 años $(16,28 \mathrm{~kg}$ ) y las patinadoras de ASODEPA (21,77 kg), registrando calificaciones regulares para la categoría Pre-Juvenil $(22,33 \mathrm{~kg})$ y mala para la categoría Juvenil $(24,67 \mathrm{~kg})$. Respecto a la diferencia de los hombres fue de 57,80\%, teniendo en cuenta que los rangos de edades no se especifican y los promedios de los deportistas de ASODEPA es de 16,67 años, mostrando así, una calificación mala respecto a las pruebas de dinamometría manual.

\section{CONCLUSIONES}

- Los antecedentes teóricos y metodológicos consultados permitieron conocer los fundamentos acerca del perfil condicional y de composición corporal en patinadores de velocidad sobre ruedas del contexto nacional e internacional. Los resultados de los estudios analizados se utilizaron como herramienta para comparar los resultados obtenidos de los patinadores de ASODEPA Bogotá. Se detectó en la revisión de la bibliografía consultada que son escasos los estudios dirigidos a esta temática en el patinaje en el contexto nacional y extranjero.

- La metodología empleada para el desarrollo de la investigación y la recolección de los resultados de las mediciones facilitaron identificar las características del perfil condicional y de composición corporal en patinadores de ASODEPA Bogotá, comprobando su pertinencia.

- Los resultados obtenidos de las mediciones del perfil condicional y la composición corporal de los patinadores de ASODEPA Bogotá, evidenciaron que los deportistas se encuentran fuera de los rangos óptimos para un patinador, 
comparados con los datos referenciados por (Burke, 2007), en aspectos como talla, el peso corporal, el porcentaje graso, este último específicamente en la categoría juvenil. A partir de los resultados de las mediciones fue posible caracterizar a los patinadores evaluados.

- La evaluación de los resultados obtenidos en las mediciones de los patinadores de ASODEPA Bogotá se efectuó mediante la comparación con resultados de otros estudios del contexto nacional e internacional, se puede afirmar que los patinadores valorados corresponden a una muestra más joven, de menor talla y peso, que los de los estudios consultados; no obstante, la muestra presenta resultados positivos en las pruebas físicas evaluadas en correspondencia con la edad, con la única excepción de los resultados del test de dinamometría que se encuentran alejados del valor promedio.

\section{REFERENCIAS BIBLIOGRÁFICAS}

Acero, J. (2002). Cineantropometría, Fundamentos y Procesos. Pamplona: Universidad de Pamplona.

Alvero, J. (2009). Protocolo de valoración de la composición corporal para el reconocimiento médico-deportivo. Documento de consenso del grupo español de cineantropometría de la federación española de medicina del deporte. Archivos de Medicina del Deporte, 166-179.

Alvero, J., Cabañas, M., Herrero, A., Martinez, L., Moreno, C., Porta, J., . . Sirvent, J. (2009). Protocolo de valoración de la composición corporal para el reconocimiento médicodeportivo. Documento de consenso del grupo español de cineantropometría de la Federación spañola de medicina del deporte. Archivos de medicina del deporte, 166-179.

Arboix, J., Aguilera, J., \& Ferrandiz, C. (2016). Resistencia Aeróbica en Hockey Patines: Análisis comparativo del Rendimiento Deportivo Efectuado con y sin Patines. Revista del Entretenimiento Deportivo.

Árias, M. (2010). La bioimpedancia como valoración del peso seco y del estado de hidratación. Diálisis y Trasplante.

Aristizábal, J., \& Restrepo, M. (2014). Validez de la bioimpedancia para estimar la composición corporal de mujeres entre los 18 y 40 años . Perspectivas en nutrición humana, 51-60.

Bermúdez, P. V. (2010). La preparación deportiva en el patinaje. efdeportes.

Bray, G., DeLany, J., Harsha, D., Volaufova, J., \& Champagne, C. (2001). Evaluation of body fat in fatter and leaner 10-y-old African American and white children: the Baton Rouge Children's Study. The American journal of clinical nutrition, 687-702.

Burke, L. (2007). Nutrición en el deporte: Un enfoque práctico. Australia: Médica Panamericana.

Carter, J. (1980). The Heath - Carter Somathotipe Method. San Diego.

CIC. (2010). Reglamento General FIRS-CIC. Portimao, Portugal: comite Internacional de Carreras- Federación Internacional de Roller Sport. 
García, J., López, J., Ogando, H., Fernández, A., Padrón, A., \& Prieto, J. (2014). Utilidad de la cineantropometría y la bioimpedancia para orientar la composición corporal y los hábitos de los futbolistas. Retos. Nuevas tendencias en Educación Física, Deporte y Recreación, 117-119.

Harre, D. (1987). Teoría del Entrenamiento deportivo.

Hernández, R., Fernández, C., \& Baptista, P. (2010). Metodología de la Investigación. Mc Graw Hill.

IDRD. (2014). Protocolo de pruebas físicas promotores de ciclovía. Bogotá, D.C..

Izquierdo, I., \& Rivero, D. (2007). Programa de Preparación del Deportista. Comisión Nacional de Patinaje Carrera y Aristicos.

Lintsi, M., Kaarma, H., \& Kull, I. (2004). Comparison of hand-to-hand bioimpedance and anthropometry equations versus dual-energy X-ray absorptiometry for the assessment of body fat percentage in 17-18-year-old conscripts. Clin Physiol Funct Imaging, 85-90.

Londoño, K., \& Bolívar, M. (2011). Comparación cinemática de los ciclos de empuje en patinadores competitivos de 11 a 17 años, utilizando una tabla deslizante y la recta en pista. tesis de grado. Pereira: Univerisidad Tecnológica de Pereira.

López, A., Izquierdo, Z., \& González, M. (2009). Determinación del gasto energético (GE) por el método factorial en patinadores cubanos de velocidad. Revista Habanera de Ciencias Médicas.

Lozano, R., \& Cárdenas, W. (2013). Análisis de la composición corporal en la preparación de los patinadores de velocidad de la selección Norte de Santander participantes en los juegos nacionales 2012. Revista Actividad Física y Desarrollo Humano.

Martínez, E. (2002). Pruebas de aptitud física. Paidotribo.

Martínez, J., \& Urdampilleta, A. (2012). La medición de la composición corporal mediante la antropometría versus bioimpedancia: sus aplicaciones en el deporte. efdeportes.

Matyk, M., \& Raschka, C. (2011). Body composition and the somatotype of european top roller speed skaters. Papers on Anthropology XX, 258-271.

Mazza, J. (2000). Antropométrica. BIOSYSTEM, 133-208.

Nahrstaedt, H., Schauer, T., Shalaby, R., Hesse, S., \& Raisch, J. (2008). Automatic control of a drop-foot stimulator based on angle measurement using bioimpedance. Artificial organs, 649-654.

Narvaéz, M. (2005). Características neurocognitivas y psicológicas de los patinadores de altos logros deportivos del departamento de antioquia, en la modalidad de carreras. Tesis de grado. Medellin, Universidad San Buenaventura.

Nunes, M., Silva, S., Monteiro, W., \& Veras, P. (2001). Estimativa da gordura corporal através de equipamentos de bioimpedância, dobras cutâneas e pesagem hidrostática. Rev Bras Med Esporte, 125-131.

Paz, B. (2016). La condición física en la aptitud deportiva del patinaje de la categoría infantil en la Federación Deportiva de Chimbonazo.

Pérez, E. (2008). Las pruebas o tests en el deporte. efdeportes.

Pietrobelli, A., Wang, Z., \& Heymsfield, S. (1998). Techniques used in measuring human body composition. Curr Opin Clin Nutr Metab Care, 439-448. 
Ramos, S. (2006). Identificación y selección de talentos para el deporte. I Encuentro Iberoamericano del Deporte Infantil y Juvenil y la Educación Física: “Un compromiso con la niñez y la juventud de Iberoamérica".

Rethaud, M. (2012). Patrones de consumo, Estado Nutricional, Trastornos Alimentarios y Consumo de suplementos en deportistas de patín carrera de Alto Rendimiento de la Ciudad de Mar del Plata. 6.

Ripka, W., Rotta, C., Ulbricht, L., \& Neves, B. (2014). Composición corporal evaluada por pliegues cutáneos y bioimpedancia en varones militares brasileños / body composition evaluated by skinfolds, bioimpedance and body mass index in adults. Revista Internacional de Medicina y Ciencias de la Actividad Física y el Deporte, 279-289.

Rolo, Y. (2011). Portal Informacional Deporte Cubano. Obtenido de Características para la selección de talentos en el deporte de Patinaje de Carreras.:

http://portal.inder.cu/index.php/recursos-informacionales/arts-cient-tec/10775caracteristicas-para-la-seleccion-de-talentos-en-el-deporte-de-patinaje-de-carreras

Sánchez, A., \& Barón, M. (2009). Uso de la bioimpedancia eléctrica para la estimación de la composición corporal en niños y adolescentes. Anales Venezolanos de Nutrición.

Shephard, R. (1996). La Resistencia en el Deporte. Barcelona: Paidotribo.

Sovak, D., \& Hawes, M. (1987). Anthropological status of international calibre speed skaters. Journal of Sports Sciences, 287-304.

Suverza, A., \& Haua, K. (2009). Manual de antropometría para la evaluación del estado nutrición en el adulto. México.

Taylor, R., Jones, I., Williams, S., \& Goulding, A. (2000). Evaluation of waist circumference, waistto-hip ratio, and the conicity index as screening tools for high trunk fat mass, as measured by dual-energy X-ray absorptiometry, in children aged 3-19. The American journal of clinical nutrition, 490-495.

WHO. (2017). World Health Organization. Obtenido de http://apps.who.int/bmi/index.jsp?introPage=intro_3.html

Yuhasz, M. (1974). Physical fitness Manual. Ontario: University of Western.

Zapata, D. (2014). Caracterización Escolar de los participantes inscritos en el Programa de Deporte Escolar 40x40 en la ciudad de Bogotá, D.C..

Zapata, D. (2014). Caracterización Escolar de los participantes inscritos en el Programa de Deporte Escolar 40x40 en la ciudad de Bogotá, D.C..

\section{WEBGRAFÍA}

Alonso, A. (2012). El entrenamiento del velocista en patinaje de velocidad. SporTraining. Obtenido de http://www.sportraining.es/2012/04/12/articulo-el-entrenamiento-delvelocista-en-patinaje-de-velocidad/\#comments

Amezqueta, I. (2007). Patín Argentino. Obtenido de Patín Argentino: http://www.exxostenerife.com/arg/articulos/00000097d10cdf80a/00000098f7008fb33.html 
Argemi, R., Mouche, M., \& Lavayén, E. (2006). isdesportsmagazine. Obtenido de isdesportsmagazine:

http://www.isde.com.ar/ojs/index.php/isdesportsmagazine/article/viewFile/31/40

Arregocés, G. (2008). Coldeportes. Obtenido de Colombia Aprende:

http://www.colombiaaprende.edu.co/html/productos/1685/w3-article-278886.html

FEDEPATIN (2017). FEDEPATIN. Obtenido de http://www.fedepatin.org.co/index.php/medalleria-kaohsiung

Garrido, A. (2010). efdeportes. Obtenido de http://www.efdeportes.com/efd141/bateria-detest-eurofit.htm

Hernández, H. (2003). efdeportes. Obtenido de Selección de talentos para el deporte, 27 años de experiencia en Cuba: http://www.efdeportes.com/efd62/talento.htm

Lozano, R., Contreras, D., \& Navarro, L. (2006). Descripción antropométrica de los patinadores de velocidad sobre ruedas participantes en los Juegos Deportivos Nacionales de Venezuela, diciembre de 2005. efdeportes.com. Obtenido de http://www.efdeportes.com/efd102/patin.htm

Lugea, C. (2010). SpeedSk8rs.com. Obtenido de Algunas Consideraciones sobre Biomecánica, Técnica y el Modelo Técnico en el Patinaje de Velocidad.:

http://www.exxostenerife.com/speedsk8/downloads/consideracionessobrebiomecanicaene Ipatinajeint.pdf

Salleg, M., \& Petro, J. (2010). efdeportes. Obtenido de http://www.efdeportes.com/efd149/aptitud-fisica-de-los-escolares.htm 